\title{
The solar eclipse and its relation with higree months
}

\author{
Abdul Rahman H. S. \\ Department of Astronomy, Collage of Science, University of Baghdad
}

E-mail: Abdrahman29@yahoo.com

\begin{abstract}
The solar eclipse occurs at short time before the crescent birth moment when the moon near any one of moon orbit nodes It is important to determine the synchronic month which is used to find Higree date. The 'rules' of eclipses are:

$\mathrm{Y}= \pm 0.997$ of Earth radius, the solar eclipse is central and $0.997<$ $|\mathrm{Y}|<1.026$ the umbra cone touch the surface of the Earth, where $\mathrm{Y}$ is the least distance from the axis of the moon's shadow to the center of the Earth in units of the equatorial radius of the Earth.

A new model have been designed, depend on the horizontal coordinates of the sun, moon, the distances Earth-Moon (rm), Earthsun (rs) and $|\mathrm{Y}|$ to determine the date and times of total solar eclipse and the geographical coordinates of spot shadow as well as the shadow diameter and the variations with time.

The results are compared with Almanac and others programs are gets a good agreements and the results show the area of eclipse shadow inversely proportional with $\mathrm{rm} / \mathrm{rs}$. The Higree month which must be begin after the solar eclipse and the relation were discussed hear.
\end{abstract}

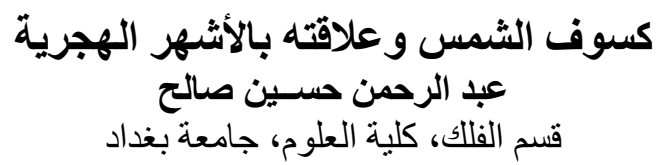

Article info. Received: Mar. 2013

Accepted: Nov. 2013

Published: Dec. 2013 solar eclipse,

lunar month.

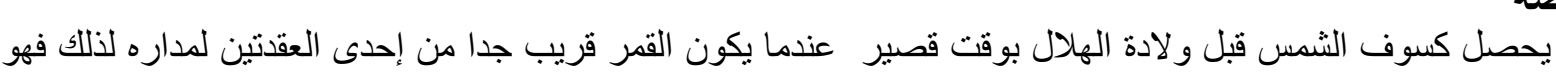

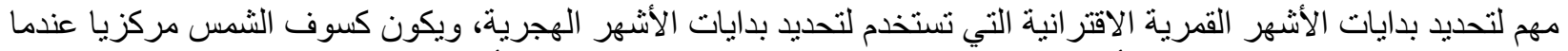
المسافة بين محور الظل ومركز الأرض

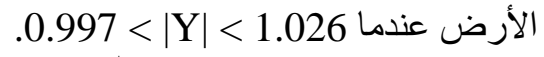

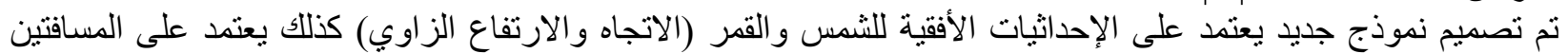

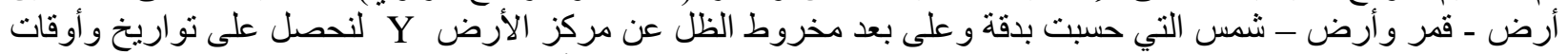

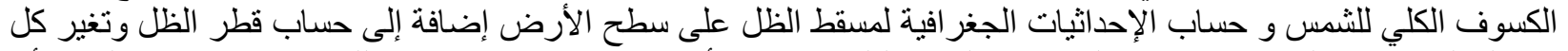

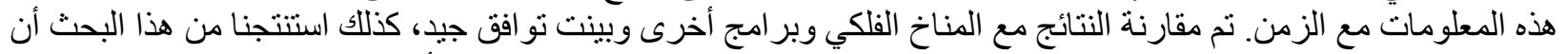
مساحة ظل الكسوف تنتاسب عكسيا مع rm / rs ، ولوحظ الكسوف دائما قبل بداية الأشهر الهجرية وتمت مناقتشة ذللك.

\section{Introduction:}

The eclipses occur when the shadows cone of the Moon falling on the Earth's surface. The solar equatorial coordinates have been change through year (The right ascension changes $(0-360)$ and the declination change $( \pm 23.445$ deg. $)$ in the geocentric equatorial system (G.E.S.) while the moon equatorial coordinates change approximately between $(0-360) \&( \pm 18, \pm$ 28 ) through sidereal month (27.53 days) $[1,2]$. 
The general important rules of eclipses [4]: (1) A lunar eclipse can only occur at full Moon and a solar eclipse at new Moon. It does n"t happens every month.

(2) At least two solar eclipses not more than five occur every year, and a maximum of three lunar eclipses in a year.

(3) Eclipses solar-lunar or lunar-solar happen pairs: (two weeks between them).

(4) The eclipses cycles of 18 years 11 days and 8 hours, called 'Saros' cycle.

(5) At the moment of greatest eclipse the Sun and Moon are either in opposition or conjunction. If the angle between the line of nodes and the Sun or Moon is less than $9^{\circ}$ a lunar eclipse occur., while if it is less than $15^{\circ}$ a solar eclipse occur.

(6) In a lunar eclipse, a maximum time of 1 hour 40 minutes, and the, partial-totalpartial, for a maximum time of 3 hours 40 minutes. The maximum time of total solar eclipse (at the equator) is 7 minutes 40 seconds and an annular eclipse can last at most for 12 minutes and 24 seconds.

To determine the solar eclipse; The coordinates of the Sun and the Moon had been calculated for any date and time. There are many astronomers calculate the coordinates of the sun and the moon, the date and time of the new moon as [3-7, 9].

The solar eclipse problem is complicated by the fact that the phases of eclipse are dependent on the observer coordinate, While the lunar eclipse all the observer see the same phase at the same instant. the total solar eclipse is occur at the sun - moon - earth angle (Elongation angle) approach zero when the moon is completely in the node at the syndic moment, the moon shadow maybe touch the Earth surface. When the moon at crescent, phase the Elongation angle is minimum and various between $0, \pm 5.2$ [5].

At total solar eclipse the solar and lunar equatorial coordinate must be the same and the umbra passes on the observer when the solar horizontal coordinates equal the lunar horizontal coordinate.

The moon's umbra shadow is about $371000 \mathrm{~km}$.in length and the earth moon distance which varies between 336000$420000 \mathrm{~km} \mathrm{[5,10]}$ that mean the moon's umbra maybe not reached the earth's surface, The total eclipses could never seen but for annular solar eclipse some of the sun disc can seen. The moons shadow diameter depend on the sun distance rs and the Earth Moon rm, which varies always with time, period through year and the shadow diameter velocity depend on moon-earth distance which varies throughout the syndic month. The moons shadow sweep across the earth depend on the moon orbital speed as a domain factor and the earth rotate as secondary factor, the shadow can be seen from a spot on the earth for the total eclipse only 2-3 minutes, A typical eclipse sweeps out a path about $10000 \mathrm{~km}$. long and about $150 \mathrm{~km}$ wide [ 2 ].

This number is $50 \%$ depend on the distances $\mathrm{rm}$ and rs. In the present work new model have been used to determine the date and time of the total solar eclipse, eclipse shadow center coordinate and shadow area on the earth surface.

\section{Calculating a total solar eclipse}

A solar eclipse is more difficult to calculate than a lunar eclipse. If the solar eclipse looked in the Astronomical Almanac a map of the world shows the path and duration of the eclipse at each point.

The solar eclipse is total when the moon disc passes on the earth -sun line this case happen only when the new moon in one of the two nodes. The solar eclipse occurs when the Earth passes the night side of Moon and the Moon shadow cone touch the Earth surface.

The solar eclipse characteristic known from the least distance from the axis of the moon's shadow to the center of the Earth in 
units of the equatorial radius of the Earth(Y) [4]. Smith P.D. see that $Y= \pm 0.9972$ the solar eclipse is central and $0.9972<|\mathrm{Y}|<$ 1.026 the umbra cone touch the surface of the Earth [5]. Others references depend on the elongation angle (the Earth-Sun - Moon angle ) must be less than 1 degree, in this work the elongation less than 1 degree and the observer - sun - moon angle is minimum than any other observers and the shadow area is more than zero.

The programs for our model are designed in Q-basic language in this big work all the years and months are chosen to limit the new Moon days, the solar eclipse type, shadow spot diameter and its geographical coordinates as the following steps:

1- Find the Julian Date (J.D.) for the new Moon with concluded all the perturbation effect on the Moon orbit [4].

2- From the J.D. compute the Julian century ( $\mathrm{T})$

T= J.D.- $2451545 / 36525$

3- $\mathrm{T}$ used to compute the ecliptic coordinates $(\beta, \lambda)$ for the Sun and Moon [4] or as in [14] take the similar results and compute the obliquity angle $\varepsilon$ $\varepsilon= \pm 23.4512-0.00256 \cos [259.18+1934.142 \mathrm{~T}$ $+0.00207 \mathrm{~T}^{2}$ ]

4- Convert the ecliptic coordinate to equatorial $(\alpha, \delta)$ for the new Moon, as in the following formula:

$\sin \delta=\sin \beta \cos \varepsilon+\cos \beta \sin \varepsilon \sin \lambda$

$\tan \alpha=(\sin \lambda \cos \varepsilon-\tan \beta \sin \varepsilon) / \cos \lambda$.

$\mathrm{H}=\mathrm{LST}-\alpha$.

LST $=$ GST - L / 15- $((\gamma / 60) / 24)$.

LST,GST: Are the local and universal sidereal time respectively.

$\mathrm{L}, \varphi$ the observer geographical coordinate (longitude and latitude).

4- Convert the equatorial coordinate to horizontal ( a , A ) for the new Moon, as in the following formula: $\sin \mathrm{a}=\sin \delta \sin \varphi+\cos \delta \quad \cos \varphi \cos \mathrm{H}(7)$ $\cos A=(\sin \delta-\sin \varphi \quad \sin a) /(\cos \varphi \quad \cos a)$

(8)

5- Calculate the Sun and Moon mean anomaly $\mathrm{M}, \mathrm{M}^{\prime}$ and the Moon mean argument of latitude $\mathrm{F}$ as the following formula [14 ]:

$\mathrm{M}=375.5256+35999.049 \mathrm{~T} \quad\}$

$\left.\mathrm{M}^{\prime}=134.96292+477198.86753 \mathrm{~T}\right\}$

$\mathrm{F}=93.27283+483202.01873 \mathrm{~T}\}$

6- Calculate the Earth - Moon and Earth Sun distances at the instate time as in the following formula [14] :

$\mathrm{rs}=\left[\begin{array}{llll}149.619 & -2.499 & \cos (\mathrm{M}) & -0.21\end{array}\right.$ $\cos (2 \mathrm{M})] 10^{\wedge} 6 \mathrm{~km}$.

$\mathrm{rm}=385000-20905 \cos \left(\mathrm{M}^{\prime}\right)-3699 \cos$ ( 2D-M' )-2956 $\cos (2 \mathrm{D})-570 \cos \left(2 \mathrm{M}^{\prime}\right)$ $+246 \cos \left(2 \mathrm{M}^{\prime}-2 \mathrm{D}\right)+205 \cos (\mathrm{M}-2 \mathrm{D})-$ $171 \cos \left(\mathrm{M}^{\prime}+2 \mathrm{D}\right) \quad-152 \cos \left(\mathrm{M}^{\prime}+\mathrm{M}-2 \mathrm{D}\right)$ $\mathrm{km}$.

where $\mathrm{D}$ is the difference between the mean longitudes of the Sun and the Moon. $\mathrm{D}=297.85027+445267.11135 \mathrm{~T}$

The Earth-Moon distance also can be calculated as in [4] that is

$r_{c}=6378.16 / \sin \pi_{c}$.

where $\pi_{\mathrm{c}}$ : The Moon parallax.

7- To find the characteristic of eclipse calculate $\mathrm{Y}$ the least distance from the axis of the moon's shadow to the center of the Earth in units of the equatorial radius of the Earth. as the following [ 4 ]: $\mathrm{S}=5.19595-0.0048 \cos \mathrm{M}+0.002 \cos 2 \mathrm{M}$ $-0.3283 \cos \mathrm{M}^{\prime}-0.006 \cos \quad\left(\mathrm{M}+\mathrm{M}^{\prime}\right)+$ $0.0041 \cos \left(\mathrm{M}-\mathrm{M}^{\prime}\right)$

$\mathrm{C}=0.207 \sin \mathrm{M}+0.0024 \sin 2 \mathrm{M}-0.039 \sin$ $\mathrm{M}^{\prime}+0.115 \sin 2 \mathrm{M}^{\prime}-0.0073 \sin \left(\mathrm{M}+\mathrm{M}^{\prime}\right)-$ $0.0067 \sin \left(\mathrm{M}^{\left.-\mathrm{M}^{\prime}\right)}+0.0117 \sin 2 \mathrm{~F}\right.$

$\mathrm{Y}=\mathrm{S} \sin \mathrm{F}+\mathrm{C} \cos \mathrm{F}$

Now at $\mathrm{Y}= \pm 0.9972$ the solar eclipse is central (+ north of earth- south of earth) at 
$0.9972<|\mathrm{Y}|<1.026$ the umbral cone touch the surface of the Earth.

8- Calculate $U$ factor as in [4].

$\mathrm{U}=0.0059+0.0046 \cos \mathrm{M}-0.0182 \cos \mathrm{M}^{\prime}$

$+0.0004 \cos 2 \mathrm{M}^{\prime}-0.0005 \cos \left(\mathrm{M}+\mathrm{M}^{\prime}\right)$

where $\mathrm{U}$ : denotes the radius of the Moons umbra cone in the Earth fundamental plane, In this work if $\mathrm{U}=+\mathrm{ve}$.

The shadow diameter (d) in the fundamental plane of Earth surface can calculate from the two large and medium triangles get:

$\mathrm{Rm} / \mathrm{Rs}=(\mathrm{X}+\mathrm{rm} /(\mathrm{X}+\mathrm{rs})$

or

$$
\mathrm{X}=(\mathrm{rs} \mathrm{Rm}-\mathrm{rm} \mathrm{Rs}) /(\mathrm{Rs}-\mathrm{Rm})
$$

where $\mathrm{Rm}$, Rs the actual radius of the Moon and the Sun are $1738 \mathrm{Km}, 696000 \mathrm{Km}$.

From the other triangles medium and small get the eclipse shadow diameter $d$ which full down on the fundamental plane:

$\mathrm{d}=\mathrm{X} \mathrm{Rm} /(\mathrm{X}+\mathrm{rm})$

9- Determine the best agreement of the equatorial and horizontal coordinate for the Sun and the Moon for the entire geographical coordinate in step 4 and print the results.

10-Return to calculate all the above steps (2 to 9) at other time ( $\mathrm{t}=\mathrm{t}+15 \mathrm{~min})$ or $\mathrm{JD}=$ JD+ $15 /(60 * 24)$ to calculate the geographical coordinate of shadow spot and it's area.

\section{Results and Discussion}

The programs which designed for this model are get the Julian date for the new Moon with concluded all the perturbation effect on the Moon orbit. The elliptical, equatorial, horizontal coordinate and the distance for the Sun and the Moon are calculated to find the date and time of total solar eclipse and position of shadow spot on the earth surface. The distances rm, rs are computed to limit the eclipse type and shadow diameter.

These results are shown in Tables (1 -11) for the years1995, 1997, 1998, 1999, 2001,
2004, 2005, 2006, 2007, 2008, 2009 and the variation of all above information with time as well as its variation with geographical coordinate, the center of shadow at best agree of coordinate, these tables contain the eclipse type, date and universal time for mid eclipse must of these results agree with Astronomical Almanac 2010 [15] that meaning our programs are right.

Table 12 shows information about some eclipses happen at the last years and comparison with other reference it's have a good agreement and some of this eclipse iare agree with observation.

Table 13 and Fig. 2 shows the variation of shadow spot diameter on the earth surface with $\mathrm{rm} / \mathrm{rs}$ for some above years. It's shows that its linear inversely proportional relation this expect relation, and because rs is slow variation but $\mathrm{rm}$ varies through month than the shadow area is inversely proportional with rm when the Earth position before the shadow cone end but rm proportion with shadow area when the Earth after see Fig. 1.

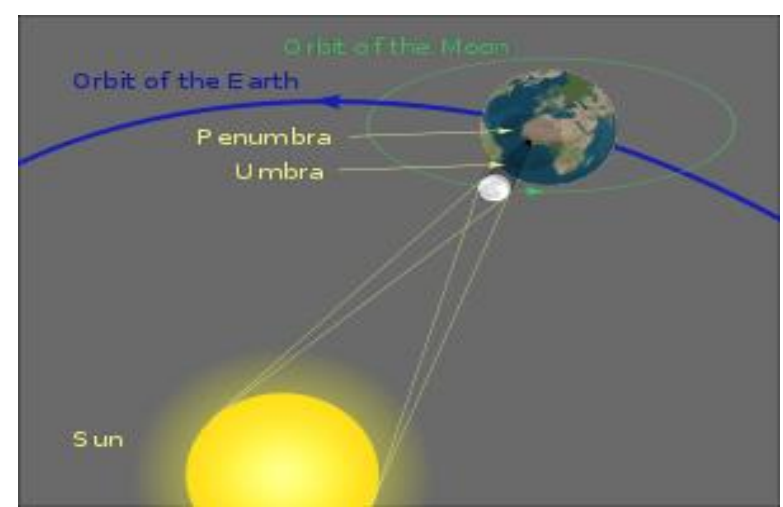

Fig. 1: The Moon shadow cone on the Earth surface and moved easterly [9].

During a central eclipse, the Moon's umbra (or ant umbra, in the case of an annular eclipse) moves rapidly from west to east across the Earth. The Earth is also rotating from west to east, but the umbra always moves faster than any given point on the Earth's surface, so it almost always appears to move in a roughly easterly 
direction across a map of the Earth see Fig.1.

The shadow diameter is plot with the ratio $\mathrm{rm} / \mathrm{rs}$ in Fig. 2 it shows a linear relation and inversely proportion that means the shadow is grater at perigee of the Moon and the beginning of lunar month is more clearly.

Table 1: YY = .3710349801 Total CENTRAL ECLPSE J.D NEW MOON 2450014.691681355 24/10/1995 4h 33min

\begin{tabular}{|c|c|c|c|}
\hline Time & Rmoon & Rsun & Sh.dim. (Km.) \\
\hline 3h 48m 59.9 & 346495.0 & 148802122.1 & 147 \\
\hline 4h 3m 59.9 & 346464.3 & 148801695.1 & 147 \\
\hline 4h 18m 59.9 & 346434.1 & 148801268.1 & 147 \\
\hline 4h 33m 59.9 & 346404.5 & 148800841.1 & 147 \\
\hline 4h 48m 59.9 & 346375.4 & 148800414.1 & 147 \\
\hline 5h 3m 59.9 & 346346.9 & 148799987.2 & 148 \\
\hline 5h 18m 59.9 & 346318.9 & 148799560.3 & 148 \\
\hline 5h 33m 59.9 & 346291.5 & 148799133.4 & 148 \\
\hline 5h 48m 59.9 & 346264.6 & 148798706.5 & 148 \\
\hline 6h 3m 59.9 & 346238.3 & 148798279.7 & 148 \\
\hline
\end{tabular}

Table 2: YY $=.934365429$ Total CENTRAL ECLPSE

J.D NEW MOON 2450516.55302816 9/3/1997SUNDAY 1h $14 \mathrm{~min}$

\begin{tabular}{|c|c|c|c|}
\hline Time & R moon & R sun & Sh.dim.( Km.) \\
\hline 0h 29m 18.5 & 358083.2 & 148541022 & 90 \\
\hline 0h 44m 18.5 & 358096.8 & 148541957.6 & 90 \\
\hline 0h 59m 18.5 & 358110.6 & 148542366.8 & 89 \\
\hline 1h 14m 18.5 & 358124.6 & 148542776.0 & 89 \\
\hline 1h 29m 18.5 & 358138.8 & 148543185.3 & 89 \\
\hline 1h 44m 18.5 & 358153.2 & 148543594.6 & 89 \\
\hline 1h 59m 18.5 & 358167.8 & 148544003.9 & 89 \\
\hline 2h 14m 18.5 & 358182.7 & 148544413.2 & 89 \\
\hline 2h 29m 18.5 & 358182.7 & 148544413.2 & 88 \\
\hline
\end{tabular}

Table 3 : YY = .2163553301 Total CENTRAL ECLPSE J.D NEW MOON 2450871.227450439 26/ 2/ 1998THURSDAY17h 25min

\begin{tabular}{|c|c|c|c|c|}
\hline Time & Rmoon & Rsun & G.sh. p. def . Eq. coord. & Dim,(Km) \\
\hline $16 \mathrm{~h} 40 \mathrm{~m} 27.3$ & 360020.1 & 148145957.6 & $\begin{array}{lllll}50 & -20 & 0.262 & 68.77 & 19.90\end{array}$ & 77 \\
\hline $16 \mathrm{~h} 55 \mathrm{~m} 27.3$ & 359999.3 & 148146326.1 & & 77 \\
\hline $17 \mathrm{~h} 10 \mathrm{~m} 27.3$ & 359978.6 & 148146694.6 & & 77 \\
\hline $17 \mathrm{~h} 25 \mathrm{~m} 27.3$ & 359958.2 & 148147063.2 & & 77 \\
\hline $17 \mathrm{~h} 40 \mathrm{~m} 27.3$ & 359937.9 & 148147431.8 & $\begin{array}{lllll}-80 & -10 & 0.273 & 42.19 & 7.92 \\
\end{array}$ & 77 \\
\hline $17 \mathrm{~h} 55 \mathrm{~m} 27.3$ & 359917.9 & 148147800.5 & $\begin{array}{lllll}-70 & 0 & 0.298 & 50.74 & 6.82 \\
\end{array}$ & 77 \\
\hline $18 \mathrm{~h} 10 \mathrm{~m} 27.3$ & 359898.0 & 148148169.2 & $\begin{array}{ccccc}-60 & 40 & 0.277 & 34.78 & 4.78 \\
\end{array}$ & 77 \\
\hline $18 \mathrm{~h} 25 \mathrm{~m} 27.3$ & 359878.3 & 148148538.0 & $\begin{array}{lllll}-50 & 60 & 0.328 & 19.89 & 18.96\end{array}$ & 77 \\
\hline $18 \mathrm{~h} 40 \mathrm{~m} 27.3$ & 359858.8 & 148148906.8 & & 77 \\
\hline $18 \mathrm{~h} 55 \mathrm{~m} 27.3$ & 359839.5 & 148149275.7 & & 77 \\
\hline
\end{tabular}


Table 4: YY = .5053345262 Total CENTRAL ECLPSE J.D NEW MOON 2451401.965079836 11/8/1999 WEDNES 11h 7min

\begin{tabular}{|c|c|c|c|c|}
\hline Time & R moon & R sun & G.sh. p. def . Eq. coord & Sh.dim (Km) \\
\hline $10 \mathrm{~h} 22 \mathrm{~m} 36.5$ & 372903.0 & 151614561.2 & $80 \quad 10 \quad 0.50122 .51257 .6$ & 55 \\
\hline $10 \mathrm{~h} 37 \mathrm{~m} 36.5$ & 372941.8 & 151614299.5 & $\begin{array}{lllll}-90 & 20 & 0.498 & 33.6 & 264.72\end{array}$ & 55 \\
\hline $10 \mathrm{~h} 52 \mathrm{~m} 36.5$ & 372980.7 & 151614037.8 & $-90 \quad 20 \quad 0.49733 .60264 .7$ & 55 \\
\hline $11 \mathrm{~h} 7 \mathrm{~m} 36.5$ & 373019.7 & 151613776. & $\begin{array}{lllll}-90 & 30 & 0.49733 .93 & 271.4 \\
\end{array}$ & 54 \\
\hline $11 \mathrm{~h} 22 \mathrm{~m} 36.5$ & 373058.8 & 151613514.1 & $-90 \quad 40 \quad 0.47633 .09278$. & 54 \\
\hline $11 \mathrm{~h} 37 \mathrm{~m} 36.5$ & 373098.0 & 151613252.1 & $\begin{array}{llllll}-90 & 50 & 0.481 & 31.15 & 284.2 \\
\end{array}$ & 54 \\
\hline $11 \mathrm{~h} 52 \mathrm{~m} 36.5$ & 373137.3 & 151612990.1 & $-80 \quad 50 \quad 0.46024 .81275 .9$ & 54 \\
\hline $12 \mathrm{~h} 7 \mathrm{~m} 36.5$ & 373176.7 & 151612728.1 & $\begin{array}{llllll}-60 & 60 & 0.486 & 13.43 & 262.9\end{array}$ & 54 \\
\hline $12 \mathrm{~h} 22 \mathrm{~m} 36.5$ & 373216.2 & 151612465.9 & $\begin{array}{lllll}-60 & 10 & 0.542 & 3.28 & 255.2\end{array}$ & 53 \\
\hline $12 \mathrm{~h} 37 \mathrm{~m} 36.5$ & 373255.8 & 151612203.7 & $\begin{array}{llllll}-70 & -10 & 0.538 & 7.55 & 253\end{array}$ & 53 \\
\hline
\end{tabular}

Table 5: YY $=.555508236$ Total CENTRAL ECLPSE J.D NEW MOON 2452081.999582159 21/6/2001 11h 57min

\begin{tabular}{|c|c|c|c|}
\hline Time & R moon & R sun & Sh.dim.( Km.) \\
\hline 11h 12m 15.1 & 341496.7 & 152038653.3 & 204 \\
\hline 11h 27m 15.1 & 341477.5 & 152038750.0 & 204 \\
\hline 11h 42m 15.1 & 341458.8 & 152038846.5 & 204 \\
\hline 11h 57m 15.1 & 341440.7 & 152038943. & 204 \\
\hline 12h 12m 15.1 & 341423.2 & 152039039.4 & 204 \\
\hline 12h 27m 15.1 & 341406.1 & 152039135.8 & 204 \\
\hline 12h 42m 15.1 & 341389.6 & 152039232. & 204 \\
\hline 12h 57m 15.1 & 341373.7 & 152039328.2 & 204 \\
\hline 13h 12m 15.1 & 341358.3 & 152039424.3 & 203 \\
\hline 13h 27m 15.1 & 341343.4 & 152039520.4 & 203 \\
\hline
\end{tabular}

Table 6: $Y Y=1.03890571$

J.D NEW MOON 2453292.6164 14/10/2004 2h 45min

\begin{tabular}{|c|c|c|c|}
\hline Time & R moon & R sun & Sh.dim.( Km.) \\
\hline 2h 0m 28.3 & 347866.5 & 149189626.0 & 145 \\
\hline 2h 15m 28.3 & 347833.8 & 149189182.8 & 145 \\
\hline 2h 30m 28.3 & 347801.7 & 149188739.6 & 145 \\
\hline 2h 45m 28.3 & 347770.1 & 149188296.4 & 145 \\
\hline 3h 0m 28.3 & 347739.1 & 149187853.2 & 145 \\
\hline 3h 15m 28.3 & 347708.5 & 149187410.0 & 146 \\
\hline 3h 30m 28.3 & 347678.5 & 149186966.8 & 146 \\
\hline 3h 45m 28.3 & 347649.0 & 149186523.6 & 146 \\
\hline 4h 0m 28.3 & 347620.0 & 149186080.5 & \\
\hline
\end{tabular}


Table 7: YY $=.352106190$ Total CENTRAL ECLPSE J.D NEW MOON 2453646.935928283 3/10/ 2005 10h 25min

\begin{tabular}{|c|c|c|c|}
\hline Time & R moon & R sun & Sh.dim. (Km.) \\
\hline 9h 55m 29.6 & 364967.9 & 149657702.8 & 70 \\
\hline 10h 10m 29.6 & 364943.3 & 149657255.0 & 70 \\
\hline 10h 25m 29.6 & 364919.2 & 149656807.2 & 70 \\
\hline 10h 40m 29.6 & 364895.5 & 149656359.5 & 70 \\
\hline 10h 55m 29.6 & 364872.4 & 149655911.7 & 70 \\
\hline 11h 10m 29.6 & 364849.7 & 149655464.0 & 71 \\
\hline 11h 25m 29 & 364827.5 & 149655016.2 & 71 \\
\hline 11h 40m 29 & 364805.9 & 149654568.4 & 71 \\
\hline 11h 55m 29.6 & 364784.7 & 149654120.6 & 71 \\
\hline
\end{tabular}

Table 8: YY = .3929685 Total CENTRAL ECLPSE J.D NEW MOON 2454000.99 22/9/2006 11h 43min

\begin{tabular}{|c|c|c|c|}
\hline Time & R moon & R sun & Sh.dim.( Km) \\
\hline 11h 13m 31.6 & 375621.2 & 150133366.5 & 26 \\
\hline 11h 28m 31.6 & 375611.1 & 150132930.4 & 26 \\
\hline 11h 43m 31.6 & 375601.6 & 150132494.3 & 26 \\
\hline 11h 58m 31.6 & 375592.5 & 150132058.1 & 26 \\
\hline 12h 13m 31.6 & 375583.9 & 150131621.9 & 26 \\
\hline 12h 43m 31.6 & 375575.9 & 150131185.7 & 26 \\
\hline 12h 58m 31.6 & 375568.3 & 150130749.5 & 26 \\
\hline 13h 13m 31.6 & 375561.3 & 150130313.2 & 26 \\
\hline
\end{tabular}

Table 9: $Y Y=1.123586$

J.D NEW MOON 2454355.031 11/9/2007 12h 43min

\begin{tabular}{|c|c|c|c|}
\hline Time & R moon & R sun & Sh.dim.( Km.) \\
\hline 11h 58m 8.7 & 370812.9 & 150589761.6 & 53 \\
\hline 12h 13m 8.7 & 370816.7 & 150589352.9 & 53 \\
\hline 12h 28m 8.7 & 370821.0 & 150588944.2 & 53 \\
\hline 12h 43m 8.7 & 370825.7 & 150588535.5 & 53 \\
\hline 12h 58m 8.7 & 370830.9 & 150588126.7 & 53 \\
\hline 13h 13m 8.7 & 370836.6 & 150587717.9 & 53 \\
\hline 13h 28m 8.7 & 370842.9 & 150587309.1 & 53 \\
\hline 13h 43m 8.7 & 370849.6 & 150586900.2 & 53 \\
\hline 13h 58m 8.7 & 370856.8 & 150586491.3 & 53 \\
\hline 14h 13m 8.7 & 370864.5 & 150586082.4 & 53 \\
\hline
\end{tabular}


Table 10: YY = .971495462 total CENTRAL ECLPSE

J.D NEW MOON 2454503.65626 7/2/2008 3h 42min

\begin{tabular}{|c|c|c|c|}
\hline Time & R moon & R sun & Sh.dim $(\mathbf{K m . )}$ \\
\hline 2h 57m 43.6 & 353750.1 & 147538467.4 & 99 \\
\hline 3h 12m 43.6 & 353731.4 & 147538725.1 & 99 \\
\hline 3h 27m 43.6 & 353713.1 & 147538982.9 & 99 \\
\hline 3h 57m 43.6 & 353695.4 & 147539240.8 & 99 \\
\hline 4h 12m 43.6 & 353678.2 & 147539498.8 & 99 \\
\hline 4h 27m 43.6 & 353661.5 & 147539756.8 & 99 \\
\hline 4h 42m 43.6 & 353645.2 & 147540014.9 & 100 \\
\hline 4h 57m 43.6 & 353629.5 & 147540273.0 & 100 \\
\hline 5h 12m 43.6 & 353614.2 & 147540531.3 & 100 \\
\hline
\end{tabular}

Table (11-a): YY = .305644197 CENTRAL ECLPSE

J.D NEW MOON 2454857.830658545 26/ 1/2009 7h 53min

\begin{tabular}{|c|c|c|c|}
\hline Time & R moon & R sun & Sh.dim.( Km.) \\
\hline 7h 8m 49.8 & 368961 & 147304455.5 & 24 \\
\hline 7h 23m 49.8 & 368953.9 & 147304636.8 & 24 \\
\hline 7h 38m 49.8 & 368947.4 & 147304818.3 & 24 \\
\hline 7h 53m 49.8 & 368941.3 & 147304999.7 & 24 \\
\hline 8h 8m 49.8 & 368935.7 & 147305181.3 & 24 \\
\hline 8h 23m 49.8 & 368930.6 & 147305363 & 24 \\
\hline 8h 38m 49.8 & 368926 & 147305544.7 & 24 \\
\hline 8h 53m 49.8 & 368921.9 & 147305726.5 & 24 \\
\hline 9h 8m 49.8 & 368918.2 & 147305908.3 & 25 \\
\hline 9h 23m 49.8 & 368915.1 & 147306090.3 & 25 \\
\hline
\end{tabular}

Table (11-b): YY $=0.077345662990$ Total CENTRAL ECLPSE J.D NEW MOON 2455034.607968499 22/7/2009 2h 33 min

\begin{tabular}{|c|c|c|c|}
\hline Time & R moon & R sun & Sh.dim.( Km.) \\
\hline 1h 48m 8.7 & 336918.7 & 151989181.6 & 224 \\
\hline 2h 3m 8.7 & 336916.8 & 151989052.0 & 224 \\
\hline 2h 18m 8.7 & 336915.5 & 151988922.3 & 224 \\
\hline 2h 33m 8.7 & 336914.8 & 151988792.6 & 224 \\
\hline 2h 48m 8.7 & 336914.7 & 151988662.8 & 224 \\
\hline 3h 3m 8.7 & 336915.2 & 151988532.9 & 224 \\
\hline 3h 18m 8.7 & 336916.2 & 151988402.9 & 224 \\
\hline 3h 33m 8.7 & 336917.8 & 151988272.9 & 224 \\
\hline 3h 48m 8.7 & 336920.1 & 151988142.8 & 223 \\
\hline 4h 3m 8.7 & 336922.9 & 151988012.6 & 223 \\
\hline
\end{tabular}


Table 12: total solar eclipse information

\begin{tabular}{|c|c|c|c|c|c|}
\hline \begin{tabular}{l}
\multicolumn{2}{c}{ Date } \\
d $\mathbf{m}$ y
\end{tabular} & $\begin{array}{c}\text { Time(U.T) } \\
\text { h. min. }\end{array}$ & $\begin{array}{l}\text { Shadow G. } \\
\text { coordinate }\end{array}$ & $\begin{array}{c}\text { Sh. iam. } \\
\text { Km. }\end{array}$ & $\begin{array}{l}\text { YY } \\
\operatorname{Re}\end{array}$ & Notes \\
\hline 24-10-1995 & $4 \quad 33$ & $60 \mathrm{~N}, 130 \mathrm{E}-130 \mathrm{w}$ & 147 & .371 & In ocean \\
\hline $9-3-1997$ & 14 & $60 \mathrm{~W}-10 \mathrm{E}$ & 90 & .934 & \\
\hline 26- 2- 1998 & $17 \quad 25$ & $20 \mathrm{~S}, 30 \mathrm{~W}-80 \mathrm{E}$ & & & \\
\hline 11- 8-1999 & 117 & $50 \mathrm{~N}, 10 \mathrm{~W}-90 \mathrm{E}$ & 55 & .505 & $\begin{array}{c}\text { Show from } \\
\text { Iraq }\end{array}$ \\
\hline $21-6-2001$ & $11 \quad 57$ & $18 \mathrm{~S}, 40 \mathrm{E}-140 \mathrm{E}$ & 204 & .555 & \\
\hline 14-10-2004 & 245 & & & 1.02 & annular \\
\hline $3-10-2005$ & $10 \quad 25$ & $130 \mathrm{E}-150 \mathrm{~W}$ & 70 & .352 & In Ocean \\
\hline $11-9-2007$ & 1243 & $100 \mathrm{~W}-20 \mathrm{E}$ & & 1.12 & Annular \\
\hline $7-2-2008$ & $3 \quad 42$ & & & & \\
\hline $26-1-2009$ & 53 & $130 W-80 W$ & 24 & .305 & In ocean \\
\hline $22-7-2009$ & 33 & $40 \mathrm{E}-150 \mathrm{E}$ & 224 & .077 & \\
\hline
\end{tabular}

Table 13: Shadow area variation with $\mathrm{rm} / \mathrm{rs}$

\begin{tabular}{|c|c|c|}
\hline JD > (days) & rm /rs & sh. Dim $(\mathbf{k m})$ \\
\hline 2451401.95 & 2.4561 & 55 \\
\hline 2451401.97 & 2.4566 & 54 \\
\hline 2451402.03 & 2.4619 & 53 \\
\hline 2454503.63 & 2.3977 & 99 \\
\hline 2454503.67 & 2.3972 & 99.5 \\
\hline 2454503.71 & 2.3967 & 100 \\
\hline 2453646.9 & 2.4388 & 70 \\
\hline 2453647 & 2.4375 & 71 \\
\hline 2453292.6 & 2.3315 & 145 \\
\hline 2453292.64 & 2.3307 & 145.5 \\
\hline 2453292.68 & 2.3299 & 146 \\
\hline
\end{tabular}

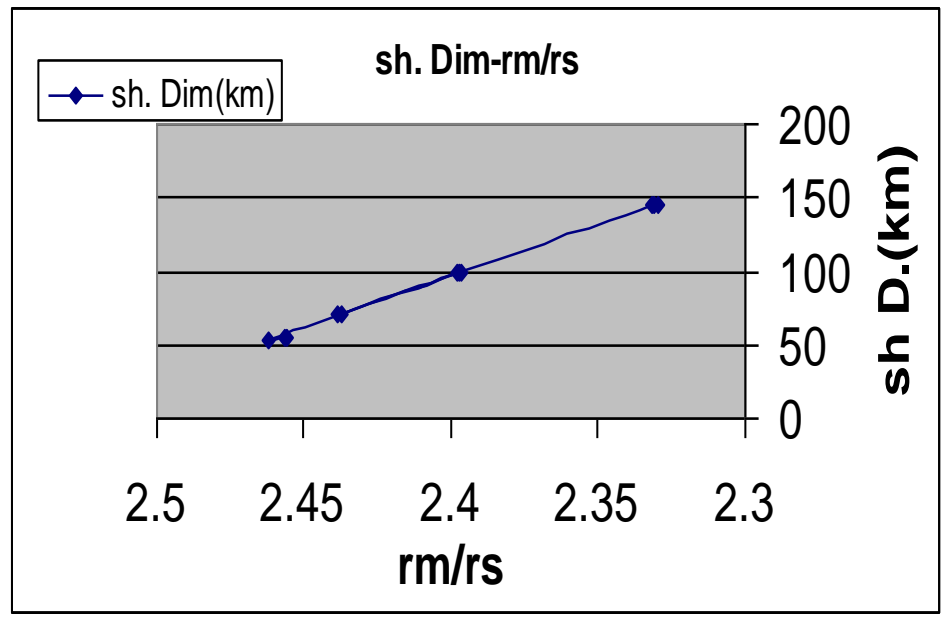

Fig.2: Shadow diameter variation with ratio $\mathrm{rm} / \mathrm{rs}$. 


\section{Conclusions}

It was noted from the figures that the eclipse was total. Comparison of our results (calculated times) with those deduced from the maps and tables of the Astronomical Ephemeris [15] shows that we are within about a quarter of an hour of the correct solar eclipse times. Even our comparatively simple method allows us to make quite accurate predictions of phenomenon. We are think this program is the big first step about the eclipse in Iraq astronomer groups.

The solar eclipse can used to determine the Hegree month where the solar eclipse moment point to the beginning of the syndic month from top centric observer, which can be used to determined Hegree month which begin at sun set after solar eclipse because this event can see from all people [2,9].

\section{References}

[1] W. M. Smart, Text book on spherical sixth edition reversed by R. M. green Cambridge Un. Press. (1977),

[2] A. H. Almohamady and S.K. Jameel (2000), Iraq Sci. J. no.2-41.

[3] M. S. Qureshi (2008), computational astronomy and the earliest visibility of lunar crescent, Ph.D. Theses, Uni. Of Karachi.
[4] J.Meeus, "Astronomical formula for calculators" second ed., 1988.

[5] P. D.Smith, "Practical astronomy with your calculator." Second ed ,1981.

[6] G.H.Kaplan, Astronomical, 2003.

[7] H. M. Alnaimy and A.H.Almohamady, I. S. J. N 2-35(1994) 580.

[8] Application, us Navel observatory website.

[9] Le Roy E. Doggett, Icarus, 107 (1994) 388-403.

[10] A. H. Almohamady and F. M. Abdula, Iraq Sci. J. 1 (2000) 42.

[11] E. Belbruno, Lunar Capture Orbits, a Method of Constructing Earth-Moon Trajectories and the Lunar GAS Mission. IN: $19^{\text {th }}$ AIAA /DGLR/JSASS International Electric Propulsion Conference, Colorado Springs, AIAA, New York, 1987.

[12] E.Belbruno, and K.Miller, Journal of Guidance, Control and Dynamics, 16, 4 (1993) 770-775.

[13] V.Chobotov, "Orbital Mechanics" 3rd Ed. AIAA, Reston, Virginia (2002).

[14] M.Oliver and G.Eberhard, Satellite Orbits, P22-85 (2001) 68-80.

[15] Astronomical Almanac of Royal. Canada, 2010. 